\title{
EL SUBREGISTRO DE ACCIDENTES DE TRABAJO EN UNA UNIDAD DE MEDICINA FAMILIAR DEL INSTITUTO MEXICANO DEL SEGURO SOCIAL SUBREGISTRO DE ACCIDENTES DE TRABAJO EN ASEGURADOS
}

\author{
PATRICIA PÉREZ MARTÍNEZ*; GUADALUPE AGUILAR MADRID**; \\ CUAUHTÉMOC ARTURO JUÁREZ PÉREZ**; FRANCISCO RAÚL SÁNCHEZ ROMÁN**
}

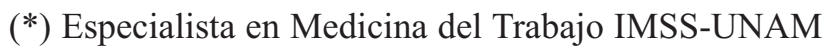

(**) Unidad de Investigación en Salud en el Trabajo CMN-Siglo XXI-IMSS

\section{RESUMEN}

Objetivo: Determinar el subregistro de los accidentes de trabajo en una Unidad de Medicina Familiar (UMFX) escogida, inicialmente atendidos en el servicio de urgencias de un Hospital General de Zona (HGZ), del Instituto Mexicano del Seguro Social (IMSS) en el año 2002.

Material y métodos: Es un estudio transversal que utilizó información de la fuente original del HGZ que otorga la primera atención médica. Se elaboró una cédula de registro con las diversas fuentes de información, efectuando confrontación, seguimiento y análisis estadístico de las mismas.

Resultados: De 6551 probables accidentes de trabajo atendidos en el HGZ, se seleccionaron 821 casos adscritos a la UMFX escogida. Se conformaron tres grupos de acuerdo con su dictamen: Grupo I= Sí de trabajo, 35.4\% (290); Grupo $\mathrm{II}=\mathrm{NO}$ de trabajo, 4.6\% (38), y el Grupo $\mathrm{III}=$ no calificados y atendidos en urgencias, 60\%(493); este último representa el subregistro de los accidentes de trabajo.

Conclusiones: El subregistro del $60 \%$ es de los más altos reportado en la literatura, lo cual representa para el IMSS, los trabajadores y el país graves repercusiones económicas y sociales no cuantificadas.

\section{PALABRAS CLAVES}

Subregistro, accidentes de trabajo, IMSS, México.

\section{ABSTRACT}

Objective: To determine the Occupational Accidents Subregistry at a selected Family Medicine Unit (FMUX) initially treated at a Mexican Institute of Social Security (IMSS) General Zone Hospital (HGZ) during the year 2002.

Materials and Methods: This is a transversal study utilizing information from the original source, the HGZ primary-care hospital-facility. We elaborated a registry permit with diverse information sources, and conducted confrontation, followup, and statistical analysis of these sources.

Results: Of 6,551 probable occupational accidents treated at the HGZ Emergency Room, we selected 821 cases ascribed to the selected FMUX. We made up three groups according these dictates: Group I = YES, occupational, 35.4\% (290); Group $\mathrm{II}=\mathrm{NO}$, occupational, $4.6 \%$ (38), and Grupo III = not qualified and treated at the Emergency Room, $60 \%$ (493); the latter group represented the occupational accidents registry.

Conclusions: The subregistry of $60 \%$ is one of the highest reported in the literature, this representing for the IMSS, its workers, and the country of Mexico severe, non-quantified, social and economic repercussions.

\section{KEY WORDS}

Subregistry, Occupational accidents, IMSS, Mexico. 


\section{INTRODUCCIÓN}

El Instituto Mexicano del Seguro Social (IMSS) afilia al $25.3 \%$ de la población económicamente activa (PEA), ${ }^{1}$ ésta es la única institución que genera y publica datos estadísticos de accidentes de trabajo (AT) en México. Los sistemas de registro de la patología legalmente reconocida como profesional (accidentes de trabajo, de trayecto y las enfermedades profesionales) han mejorado; sin embargo, el proceso continúa teniendo algunas limitaciones administrativas; además, existen causas externas no estudiadas, las cuales dan como resultado un subregistro escasamente cuantificado. ${ }^{2}$

Desde la década de los ochenta, los AT han mantenido un constante descenso en su frecuencia, ya que pasaron de una tasa de 7.2/100 en 1985 a 2.3/100 en el 2005. ${ }^{3}$ Sin embargo, esta disminución no se puede concebir sin pensar en un probable subregistro, pues nuestros resultados son poco creíbles al compararlos con algunos países desarrollados; por ejemplo: en el periodo de 1991 a 2002, nuestras tasas fueron de 5.5 a $2.5 / 100$, los cuales son similares o menores a Italia $(6.2$ a $4.0 / 100)$, España $(6.7$ a 7.1/100) y Suiza (4.2 a $2.5 / 100){ }^{4}$

Esta disminución está influida por diferentes causas, que tampoco han sido estudiadas, como la modernización de los procesos productivos, una mayor capacitación a los trabajadores en la prevención de AT y la implementación de programas preventivos; asimismo, el ocultamiento de los AT por parte de las empresas, con las siguientes modalidades: contratación, por parte de las empresas, de servicios médicos privados que atienden a los trabajadores que sufren un AT, absorbiendo el gasto total de su atención y de sus días de incapacidad. En una segunda conducta, la empresa "permite" que el IMSS atienda al trabajador accidentado y niega su reconocimiento al no continuar con los trámites administrativos para su registro (llenado del formato ST-1), por lo que el IMSS absorbe los costos de atención médica e incapacidades temporales. Otra forma de evadir su responsabilidad en el pago de la prima de riesgos de trabajo es registrando los accidentes de trabajo como de trayecto, cuyos costos de atención médica y secuelas son absorbidos totalmente por el IMSS, de acuerdo con la Ley del Seguro Social,. De estas causas mencio- nadas, no se han estudiado ni evaluado su magnitud y el peso de cada una en la determinación del subregistro. $^{2}$

En un estudio realizado en el IMSS para cuantificar el subregistro de los AT en los diversos estados de la república, se reportó un promedio nacional del $26.3 \%$ de subregistro, con un rango de 0 a $68 \%{ }^{2}$ Otros reportes han evaluado el fenómeno del subregistro; sus resultados son los siguientes: en Los Cabos, San Lucas, Baja California Sur, 48\% ${ }^{5}$ en Acapulco, Guerrero, 35.2\% ${ }^{6}$; Macías y Pérez (1999), en Jalisco, encontraron $53.4 \%$ de subregistro, ${ }^{7}$ entre otros estudios.

El propósito de este estudio fue determinar la prevalencia del subregistro de los accidentes de trabajo (AT), utilizando fuentes de información secundarias, confrontando los diversos instrumentos de registro e información, en una Unidad de Medicina Familiar del IMSS, en el Distrito Federal en el periodo de un año.

\section{MATERIAL Y MÉTODOS}

En el presente estudio se definió como caso de subregistro de AT a todo aquel accidente que recibió la atención médica inicial en el servicio de urgencias del Hospital General de Zona (HGZ), y que el trabajador haya referido que la lesión ocurrió en el trabajo, y que no haya sido calificado como tal por los Servicios de Salud en el Trabajo (SST) de la Unidad de Medicina Familiar (UMFX) donde se realizó el presente estudio, sin su correspondiente ingreso al sistema de registro de riesgos de trabajo SUI55/ ST-5. ${ }^{5}$

El IMSS cuenta con 37 delegaciones administrativas en el ámbito nacional. Se escogió la Delegación 4 del Distrito Federal (D4), con un Hospital General de Zona, y de sus respectivas siete UMF de influencia, también se eligió solo una. Los motivos de selección fueron: por las facilidades brindadas para la revisión de los formatos de atención médica inicial (ST-4-30-8) y de calificación de accidentes de trabajo (ST-1) y su confrontación con los registros de la memoria estadística del IMSS en $2002 ;^{3}$ en esta D4, estaban afiliadas 27,558 empresas, lo que representa el 3.4\% del total nacional de accidentes de trabajo, con 439,187 (3.6\%) trabajadores bajo el seguro 
de riesgos de trabajo. En ese mismo año, en la D4 se presentaron 21,569 casos de riesgos de trabajo, lo que representa el 5.6\% del total nacional de RT), de los cuales el 70.7\% (15 323) corresponden solo a AT $\mathrm{y}$, de éstos, 506 (3.86\%) generaron incapacidades permanentes (IPP), es decir, una tasa de 33 IPP por cada 1000 accidentes de trabajo. ${ }^{8}$

\section{Fuentes de Información}

Para lograr nuestro objetivo de identificar los casos de probables AT atendidos en el servicio de urgencias o en la consulta externa de primero y segundo nivel de atención del IMSS, se consultaron las siguientes fuentes de información:

- Hojas de atención de urgencias traumatológicas del Hospital general de Zona (Formato 1), de las cuales se obtuvieron la ficha de identificación y especificaciones del accidente de trabajo, diagnóstico, fecha y hora de los accidentes, edad, la forma de arribo al hospital y lugar de procedencia; número de registro, afiliación, UMF, diagnóstico, atención otorgada y médico tratante.

- Reporte de la División Técnica de Información y Estadística en Salud (forma SUI55/ST5), ${ }^{5}$ para contrastar los accidentes de trabajo de la D4 y UMFX contra los accidentes terminados, que son los que ingresan al sistema de información SUI55/ST5 (Formato de registro de riesgos de trabajo ocurridos y terminados y casos de invalidez) del IMSS en el 2002.

- Formatos ST-1 (Hoja de aviso para calificar probable riesgo de trabajo) del Servicio de Salud en el Trabajo de la UMFX. De estos formatos se recolectaron: número de afiliación, género, salario, tipo de riesgo, accidente, diagnóstico, fecha de calificación, médico dictaminador. Para poder identificar en el Servicio de Salud en el Trabajo de la UMFX los casos calificados como "sí" de trabajo y "no" de trabajo.

- Sistema de Información Nacional de Derechos y Obligaciones (SINDO). ${ }^{10}$ De este sistema se obtuvieron el consultorio asignado a los trabajadores para localizar los expedientes de los casos no calificados por el SST, así como la vigencia de derechos de los asegurados.
- Reporte semanal de ST-5 del SST de la UMFX. Aquí se reviso el informe semanal de casos terminados de riesgos de trabajo, para identificar los casos que tenían requisitado el formato ST-5 y cuáles de éstos sí entraron al sistema de información SUI55/ST5 de la D4.

- Expediente Clínico del Consultorio en la UMFX. Permitió conocer el diagnóstico inicial y final y los días de incapacidad generados por cada accidente de trabajo; asimismo, verificar el seguimiento y resolución del AT en el Servicio de Salud en el Trabajo.

Con la información recabada y confrontada se conformaron tres grupos: Grupo I = calificados como SÍ de trabajo, Grupo II = calificados como NO de trabajo, y el grupo III =, los que no concluyeron el trámite de calificación del AT, y que definimos con caso de subregistro.

\section{Plan de análisis}

Se generó una sola base de datos con todas las fuentes de información recopiladas y se realizó el análisis estadístico; se calcularon medidas de tendencia central (media, mediana y moda) y de dispersión (rango, desviación estándar y varianza), así como análisis de varianza, diferencias de medias (ANOVA) y proporciones. Asimismo, un análisis de frecuencias simples de los diagnósticos y su agrupamiento. Para analizar los datos, se utilizó el paquete estadístico STATA V. 8.

\section{RESULTADOS}

En un Hospital General de Zona (HGZ) de la D4, seleccionado para el presente estudio durante el periodo de del $1^{\circ}$ de enero al 31 de diciembre de 2002, se recolectaron 6551 hojas de atención de Urgencias de Traumatología por probable AT; de estos casos, 4,505 (68.7\%) fueron hombres y 2,046 (31.3\%) mujeres, distribuidos en siete unidades de Medicina Familiar (UMF) del sistema. La UMF con mayor porcentaje de casos de probables AT de la D4 fue la UMF "A", con 2,261 (34.5\%). De las anteriores siete UMF, que corresponden al HGZ seleccionado, se eligió la denominada Unidad de Medicina Familiar X (UMFX), donde se reportaron en 2002 un total de 821 casos de accidentes de trabajo, de los cuales se corroboró su adscripción. De estos 821 AT, 
$73.4 \%$ (603) fueron hombres y $26.6 \%$ (218) mujeres. Se confrontaron los números de afiliación de los casos de AT atendidos en el HGZ, que correspondían a la UMFX con la base de datos de la División Técnica de Información y Estadística en Salud proveniente de los formatos SUI55/ST5 del 2002. ${ }^{9}$ Sólo dos $(3.17 \%)$ del total de casos se encontraron registrados en esa base de datos que reporta los riesgos de trabajo terminados. De igual manera, se cotejaron estos casos con la base de datos empleando el Sistema de Información Nacional de Derechos y Obligaciones (SINDO), ${ }^{10}$ para identificar el consultorio de medicina familiar asignado en la UMFX, así como con los archivos del SST de la UMFX de las ST-1 calificadas como sí de trabajo en el año 2002. Se encontraron 290 (35.4\%) casos, de los cuales solo $58.3 \%$ (169) tenían ST-5 (registro de riesgos de trabajo) y el alta; el resto no contaba con ellos ni una nota que refiriera la evolución del paciente.

La base de datos quedó conformada con 821 casos distribuidos en los tres grupos definidos: Grupo I = calificados como Sí de trabajo, 290 (35.4\%); Grupo II = calificados como NO de trabajo, $38(4.6 \%)$, y el grupo III = los que no concluyeron el trámite de calificación del AT y que cumplen la connotación de caso subregistrado, con 493 casos
(60\%) (Cuadro I). Los años de antigüedad laboral promedio $(\mathrm{DE}=$ desviación estándar) y [rango] fueron de 3.5 (6.1) [0.01 a 42] años. La edad promedio fue de 33 años (12) [16 a 71]; los días de incapacidad promedio generados por cada caso fueron de 14 días (29) [0 a 358] (Cuadro I). En el análisis por grupos para ver diferencia de medias se observó que edad y antigüedad no fueron estadísticamente significativas. Sin embargo, los días de incapacidad fueron 22.8 días en promedio por caso para el grupo I, calificados como AT; para el grupo III, 15 días en promedio, calificados como enfermedad general; el grupo II no había reportado incapacidades (Cuadro I). Con respecto al salario promedio para los del grupo I, fue de $\$ 143.00$ pesos $(\mathrm{DE}=\$ 136.00)$ y un rango de $\$ 40.00$ a $\$ 870.00$ pesos; en el grupo II, fue de $\$ 157$ pesos $(\mathrm{DE}=\$ 194)$ y un rango de $\$ 42$ a $\$ 879.00$ pesos; en el grupo III no estaba reportado el salario (Cuadro I).

En los tres grupos hubo predominio de los hombres 73.4\% (603), (Cuadro II). De los 493 (60\%) casos que representan el subregistro, se revisaron los expedientes clínicos de su consultorio familiar; sólo se encontraron 249 (50.5\%), de los cuales $122(49 \%)$ tenían la hoja de atención de urgencias de traumatología (formato 1); sin embargo, no existía ninguna

\begin{tabular}{|c|c|c|c|c|c|c|c|c|c|c|c|c|c|c|c|c|c|c|c|c|}
\hline \multirow[t]{2}{*}{ Variable } & \multicolumn{5}{|c|}{ Grupo I } & \multicolumn{5}{|c|}{ Grupo II } & \multicolumn{5}{|c|}{ Grupo III } & \multicolumn{5}{|c|}{ Total } \\
\hline & $\mathrm{n}$ & media & Mdn & $\mathrm{DE}$ & $\begin{array}{l}\text { Ran } \\
\text { go }\end{array}$ & $\mathrm{n}$ & media & Mda & $\mathrm{DE}$ & $\begin{array}{l}\text { Ran } \\
\text { go }\end{array}$ & $\mathrm{n}$ & Media & Mda & $\mathrm{DE}$ & $\begin{array}{l}\text { Ran } \\
\text { go }\end{array}$ & $\mathrm{n}$ & media & Mda & $\mathrm{DE}$ & $\begin{array}{c}\text { Ran } \\
\text { go }\end{array}$ \\
\hline $\begin{array}{l}\text { Edad } \\
\text { (años) }\end{array}$ & 290 & 34 & 29 & 12.2 & $\begin{array}{l}16- \\
71\end{array}$ & 38 & 31 & 29 & 11 & $\begin{array}{l}17- \\
58\end{array}$ & 493 & 33 & 30 & 12 & $\begin{array}{l}16- \\
68\end{array}$ & 822 & 33 & 30 & 12 & $\begin{array}{l}16- \\
71\end{array}$ \\
\hline $\begin{array}{l}\text { Incapacidad } \\
\text { (dias) }\end{array}$ & 277 & 22.8 & 14 & 29 & $\begin{array}{c}1- \\
358\end{array}$ & 0 & - & - & - & - & 77 & 15 & 7 & 25 & $\begin{array}{c}2- \\
151\end{array}$ & 278 & 14 & 23 & 29 & $\begin{array}{c}0- \\
358\end{array}$ \\
\hline $\begin{array}{l}\text { Antigüedad } \\
\text { (años) }\end{array}$ & 199 & 3.58 & 1 & 6.2 & $\begin{array}{l}0.01 \\
-42\end{array}$ & 33 & 3.57 & 0.8 & 6.07 & $\begin{array}{l}0.01 \\
-24\end{array}$ & 0 & - & - & - & - & 232 & 3.57 & 1 & 6.1 & $\begin{array}{c}0.0- \\
42\end{array}$ \\
\hline
\end{tabular}

$\begin{array}{llllllllllllllllllllll}\begin{array}{l}\text { Salario } \\ (\$)\end{array} & 220 & 143 & 99 & 136 & 40- & 33 & 157 & 102 & 194 & 42- & 0 & - & - & - & - & 253 & 145 & 100 & 145 & 40- \\ 879\end{array}$

Fuente: Archivo del HGZ, Serv. Urgencias traumatología, ST-1, Informes semanales de ST-5, Expedientes clínicos UMF.

Para el grupo I las incapacidades son por riesgo de trabajo y para el grupo III por enfermedad general

Cuadro I. Distribución de edad, antigüedad, salario y días de incapacidad de acuerdo con los grupos de estudio de la UMF X atendidos en HGZ, IMSS. 2002 
nota del medico familiar del probable AT ni de su envío a los SST y tampoco de su calificación como AT. De los otros 244 (49.5\%) casos, en 127 (52\%) se encontraba el expediente sin formato 1 , y no había nota médica que refiriera el probable AT. En 244 (49.5\%) casos no se encontraron los expedientes; de estos últimos, 17 (2.1\%) no tenían consultorio asignado, por lo que no había forma de localizar el expediente y $23(2.8 \%)$ casos no aparecían vigentes en el SINDO (Cuadro II).

De los 821 casos registrados inicialmente como AT, según la forma de arribo al servicio de urgencias, el $64.7 \%$ (532) venía de su trabajo y el $28.3 \%$ (232) de su domicilio. A 791 (96.2\%) de los atendi- dos se les dio de alta y fueron enviados a la UMFX para continuar la atención; a $42(5.1 \%)$ se les dio cita para algún servicio de especialidad, por requerir subsecuencia en el tratamiento.

En el análisis de varianza entre los tres grupos para observar diferencias, los resultados mostraron que no hubo diferencias estadísticamente significativas para la antigüedad en el empleo, 3.6 años $(\mathrm{DE}=6.2)(p=0.8901)$. El promedio de edad entre géneros en los tres grupos fue de 32.7 años $(\mathrm{DE}=$ 11.9) para los hombres y de 34.5 años $(\mathrm{DE}=11)$ para las mujeres; la diferencia fue estadísticamente significativa $(p=0.05)$.

\begin{tabular}{|c|c|c|c|c|c|c|c|c|}
\hline \multirow{3}{*}{$\begin{array}{l}\text { Variable } \\
\text { Género }\end{array}$} & \multicolumn{2}{|c|}{ Grupo I* } & \multicolumn{2}{|c|}{ Grupo II ${ }^{\ominus}$} & \multicolumn{2}{|c|}{ Grupo III ${ }^{\varnothing}$} & \multicolumn{2}{|c|}{ Total } \\
\hline & \multicolumn{2}{|c|}{$\mathrm{n}=290(35 \%)$} & \multicolumn{2}{|c|}{$\mathrm{n}=38(5 \%)$} & \multicolumn{2}{|c|}{$\mathrm{n}=493(60 \%)$} & \multicolumn{2}{|c|}{$\mathrm{n}=821$} \\
\hline & No & $\%$ & No. & $\%$ & No. & $\%$ & No. & $\%$ \\
\hline Hombres & 218 & 75.1 & 26 & 67 & 359 & 73 & 603 & 73.4 \\
\hline Mujeres & 72 & 24.9 & 12 & 32 & 134 & 24 & 218 & 26.6 \\
\hline \multicolumn{9}{|l|}{ Forma de arribo } \\
\hline Trabajo & 226 & 77.9 & 24 & 61.5 & 282 & 57 & 532 & 64.7 \\
\hline Domicilio & 44 & 15.2 & 12 & 33.3 & 176 & 36 & 232 & 28.3 \\
\hline \multicolumn{9}{|l|}{ Región anatómica } \\
\hline Miembro torácico & 122 & 42.7 & 7 & 18.4 & 172 & 35 & 301 & 36.6 \\
\hline Miembro pélvico & 73 & 25.1 & 10 & 26.3 & 141 & 29 & 224 & 27.2 \\
\hline Columna & 53 & 18.2 & 18 & 47.4 & 101 & 21 & 172 & 21 \\
\hline Cabeza / cara & 24 & 8.2 & 1 & 2.7 & 36 & 0.3 & 61 & 7.4 \\
\hline Tórax & 7 & 2.41 & - & - & 19 & 3.8 & 26 & 3.2 \\
\hline Sin consignar & 3 & 1 & - & - & 11 & 2.2 & 14 & 1.7 \\
\hline Policontundido & 5 & 1.72 & - & - & 9 & 1.8 & 14 & 1.7 \\
\hline Cintura escapular & 1 & 0.34 & 1 & 2.7 & 2 & 0.4 & 4 & 0.48 \\
\hline Abdomen & 2 & 0.69 & 1 & 2.7 & 2 & 0.4 & 5 & 0.61 \\
\hline \multicolumn{9}{|l|}{ ST-5 } \\
\hline $\mathrm{Sin} \mathrm{ST}-5$ & 121 & 41.7 & 38 & 100 & 493 & 100 & 652 & 79.4 \\
\hline Con ST - 5 & 169 & 58.3 & - & - & - & - & 169 & 20.6 \\
\hline \multicolumn{3}{|l|}{ Expediente UMF } & \\
\hline \multicolumn{3}{|l|}{ Sin expediente } & \multicolumn{5}{|c|}{$244 \quad 49.5$} & \\
\hline \multicolumn{3}{|l|}{ Con expediente } & & & 249 & 50.5 & & \\
\hline Vigencia & & & \multicolumn{6}{|c|}{$n=493$} \\
\hline \multicolumn{3}{|l|}{ Con vigencia } & \multicolumn{3}{|r|}{470} & \multicolumn{3}{|l|}{95.3} \\
\hline \multicolumn{3}{|l|}{ Sin vigencia } & \multicolumn{3}{|c|}{23} & \multicolumn{3}{|l|}{4.7} \\
\hline \multicolumn{9}{|c|}{ Fuente: Archivo del HGZ. Serv. Urgencias traumatología, ST-1, Informes semanales de ST-5, Expedientes clínicos UMF № 46.} \\
\hline *Grupo I Los calit & s como $\mathbf{S}$ & dentes de & & & & & & \\
\hline $\begin{array}{ll}\oslash_{\text {Grupo II. }} & \text { Los calif } \\
\varnothing \text { Grupo III } & \text { Los no c }\end{array}$ & dos y ate & identes d & STRO) & & & & & \\
\hline
\end{tabular}

Cuadro II. Características de los accidentes reclamados como de trabajo en urgencias del HGZ y que corresponden a la UMFX. 2002 
El 68.3\% (560) acudió al servicio de urgencias en el turno vespertino. La región anatómica más afectada por grupo fue: el miembro torácico, $42.7 \%$ (122), en el grupo I; la columna vertebral, $47.4 \%$ (18), en el grupo II; y también el miembro torácico, 35\% (172), en el grupo III, seguido del miembro pélvico, 224 (27.2\%); finalmente, columna, 172 (21\%.) (cuadro II).

El promedio y la (DE) de edad en cada una de las lesiones según región anatómica fueron: miembro torácico, 32.8 años $(\mathrm{DE}=11.5)$; miembro pélvico, 33 años $(\mathrm{DE}=11.9)$; y para columna, 32 años $(\mathrm{DE}=10.7)$. Se encontró una diferencia estadísticamente significativa entre los grupos $(p=0.0129)$.
De acuerdo con la CIE-10, los tres principales padecimientos más frecuentes del grupo I y III por región anatómica fueron: en miembro torácico, la contusión de dedos de la mano sin daño a uñas, 24 $(20.3 \%)$ y $36(20.9 \%)$ casos, respectivamente; para miembro pélvico, esguince de tobillo, $23(31.5 \%)$ y $34(24 \%)$ casos; finalmente, para la contusión de región lumbosacra, $26(49 \%)$ y $58(57.4 \%)$ casos, respectivamente. (Cuadro III.)

\section{DISCUSIÓN}

Nuestros resultados muestran un subregistro del $60 \%$ (493), que coinciden con lo reportado en otros estudios realizados también en el IMSS; se conside-

\begin{tabular}{|c|c|c|c|c|}
\hline \multicolumn{2}{|l|}{ Sí de trabajo } & \multicolumn{3}{|c|}{ Subregistro } \\
\hline Miembro torácico & Frec. & $\%$ & Miembro torácico & Frec. \\
\hline 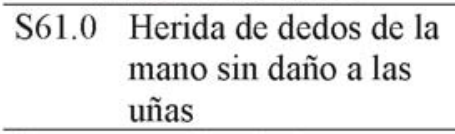 & 24 & 20.3 S60.X & $\begin{array}{l}\text { Traumatismo } \\
\text { superficial de la } \\
\text { muñeca y de la mano }\end{array}$ & $36 \quad 20.9$ \\
\hline $\begin{array}{l}\text { S61.X Heridas de la muñeca } \\
\text { y de la mano }\end{array}$ & 13 & 10.6 S61.X & $\begin{array}{l}\text { Heridas de la muñeca } \\
\text { y de la mano }\end{array}$ & 15.6 \\
\hline Esguince de muñeca & 12 & $9.8 \quad S 61.0$ & $\begin{array}{l}\text { Herida de dedos de la } \\
\text { mano sin daño a las } \\
\text { uñas }\end{array}$ & 7.5 \\
\hline Miembro pélvico & Frec. & $\%$ & Miembro pélvico & Frec. \% \\
\hline S80.0 Contusión de rodilla & 13 & $17.8 \mathrm{~S} 90.1$ & $\begin{array}{l}\text { Contusión de dedos } \\
\text { del pie sin daño a las } \\
\text { uñas }\end{array}$ & 107 \\
\hline S83.4 Esguince de rodilla & 4 & 5.4 S90.X & $\begin{array}{l}\text { Traumatismo } \\
\text { superficial del tobillo } \\
\text { y del pie }\end{array}$ & 10 \\
\hline S93.4 Esguince de tobillo & 23 & $31.5 \mathrm{~S} 93.4$ & Esguince de tobillo & 34 \\
\hline Columna & Frec. & $\%$ & Columna & Frec. $\%$ \\
\hline S13.4 Esguince cervical & 14 & $26.4 \mathrm{~S} 13.4$ & Esguince cervical & $8 \quad 7.9$ \\
\hline $\begin{array}{l}\text { Contusión de región } \\
\text { lumbosacra y de } \\
\text { pelvis }\end{array}$ & 26 & $\mathrm{~S} 23.3$ & $\begin{array}{l}\text { Esguince de columna } \\
\text { torácica }\end{array}$ & 6.9 \\
\hline $\begin{aligned} \text { T06.X } & \text { Traumatismos } \\
& \text { diferentes partes del } \\
& \text { cuerpo no clasificados }\end{aligned}$ & 3 & $5.6 \mathrm{~S} 30.0$ & $\begin{array}{l}\text { Contusión de región } \\
\text { lumbosacra y de } \\
\text { pelvis }\end{array}$ & 57.4 \\
\hline
\end{tabular}

Cuadro III. Los tres diagnósticos más frecuentes según la CIE-10 y grupo de calificación 
ran como de los más altos. En los estudios de Salinas, ${ }^{2}$ Jaramillo, ${ }^{5}$ Acosta, ${ }^{6}$ Macías $^{7}$ y García, ${ }^{11}$ se reporta un porcentaje de subregistro que oscila en un rango de 0 a $60 \%$; el único que reporta un subregistro de $77.9 \%$, mayor al nuestro, es Franco y col., en el Estado de México ${ }^{12}$ (Cuadro IV). Los estados con porcentajes similares al nuestro son Tabasco $(60 \%)$, Morelos (68\%) y Michoacán (51\%). ${ }^{2}$

Es posible que la disminución en las tasas de AT de 1985 a 2005 (figura 1) se pudiera explicar por cambios en los procesos productivos de algunas empresas. Sin embargo, consideramos que el mayor peso lo tiene el subregistro en cualquiera de las modalidades ya señaladas, una de las cuales medimos en este estudio. Esto lo afirmamos porque, de ser cierta la tendencia a la baja de las tasas de AT, también debieran verse reflejadas a la baja las incapacidades permanentes parciales; y no es así, pues éstas tienden a incrementarse en el periodo de 1997 $(32 / 1000)$ a $2005(39.2 / 1000))^{3}$

El promedio de días de incapacidad observado en los casos no registrados fue de 15 , con un mínimo y un máximo de dos a 151 días, casi cuatro veces más al señalado por Colunga, con cuatro días promedio en los casos no reclamados, y un rango de uno a 17 días. Es posible que la gravedad de los AT de nuestro estudio sea mayor a la de los estudiados por Colunga. ${ }^{13}$

La vigencia de derechos en los casos de subregistro en nuestro estudio fue de $4.7 \%$ (23), similar al reportado por Salinas y col. ${ }^{2}$ con un $6 \%$. En todos los estudios señalados, incluido el nuestro, predominan los casos no registrados de AT en el sexo masculino. Nuestros datos muestran al 34.8\% (172) de los casos de subregistro, con lesión en miembro torá-

\begin{tabular}{|c|c|c|c|}
\hline Autor y año & Número y lugar & $\begin{array}{l}\text { Tiempo } \\
\text { del } \\
\text { estudio }\end{array}$ & $\begin{array}{c}\% \\
\text { subregistro }\end{array}$ \\
\hline $\begin{array}{l}\text { Franco y col. } \\
(1994)^{14}\end{array}$ & $\begin{array}{c}\text { N265= casos } \\
\text { UMF de la subdelegación Naucalpan }\end{array}$ & Un año & $77.9 \%$ \\
\hline $\begin{array}{l}\text { Salinas y col. } \\
(2003)^{2}\end{array}$ & $\begin{array}{c}\mathrm{n}=8,013 \text { casos de } 27(72.9 \%) \text { de las } 37 \\
\text { delegaciones del sistema. }\end{array}$ & Un mes & $26.3 \%$ \\
\hline $\begin{array}{l}\text { García y col. } \\
(2003)^{13}\end{array}$ & $\begin{array}{c}\mathrm{n}=4269 \text { total, donde } 2883 \text { fueron AT y } 967 \text { de } \\
\text { trayecto. } \\
\text { De la UMF No. 61, Edo. Méx. Pte. }\end{array}$ & Un año & $50 \%$ \\
\hline $\begin{array}{l}\text { Macías y col. } \\
(1999)^{7}\end{array}$ & $\begin{array}{c}\mathrm{n}=561 \text { casos atendidos como RT en Urgencias } \\
\text { HGR No. } 46 \text {, Guadalajara. }\end{array}$ & $\begin{array}{l}\text { Tres } \\
\text { meses }\end{array}$ & $53.4 \%$ \\
\hline Acosta $(1998)^{6}$ & $\begin{array}{l}\mathrm{n}=1352 \text { casos } \\
\text { Delegación Gro. }\end{array}$ & Un año & $35.2 \%$ \\
\hline $\begin{array}{l}\text { Colunga y col. } \\
(1998)^{15}\end{array}$ & $\begin{array}{c}n=73 \text { casos } \\
\text { UMF 14, Quintana Roo }\end{array}$ & Un mes & $63 \%$ \\
\hline $\begin{array}{l}\text { Jaramillo y col. } \\
(1997)^{5}\end{array}$ & $\begin{array}{c}n=1810 \\
\text { HGZ subzona 26 BCS. }\end{array}$ & Un año & $48.45 \%$ \\
\hline $\begin{array}{l}\text { Pérez y col., (2004) } \\
\text { (Estudio actual) }\end{array}$ & $\begin{array}{c}\mathrm{N}=6551 \text { y } \mathrm{n}=821 \\
\text { HGZ y UMF, respectivamente }\end{array}$ & Un año & $60 \%$ \\
\hline
\end{tabular}

Cuadro IV. Comparación de la prevalencia de subregistro en diferentes estudios en México 
cico (manos y dedos), similar a la misma región anatómica lesionada que se reporta en los casos calificados como sí de trabajo, como el de Montes de Oca y col. ${ }^{14,15}$

El presente estudio nos permitió identificar algunas inconsistencias en el proceso de atención y calificación de los accidentes de trabajo, como son: el flujo de la información entre las unidades médicas de atención, es decir la nota medica inicial (formato 1) no es enviada a los Servicios de Salud en el Trabajo (SST) de las unidades de adscripción de los trabajadores. No se está utilizando el formato Informe Médico Inicial de Probable riesgo de trabajo 4-30-8. Por lo tanto, las notas médicas que llegan a los SST no son todos los casos atendidos en los HGZ.

Este estudio muestra sólo el subregistro de AT en una UMX. Es conveniente investigar la repercusión negativa en los trabajadores y la Institución, en los ámbitos económico y social, rubros que no fueron evaluados, y que se verán reflejados en la pérdida de las prestaciones en especie y económicas a las que tienen derecho los trabajadores, de acuerdo con los artículos 487 de la Ley Federal del Trabajo ${ }^{16}$ y 56 y 58 de la Ley del Seguro Social. ${ }^{17}$ Por lo que estamos ciertos de que el proceso de valoración y calificación de los riesgos de trabajo debe revisarse y modificarse, con el fin de corregir las desviaciones encontradas.

El impacto de los cambios en la normatividad institucional beneficiará a la institución, a los trabajadores afiliados a la misma y a las empresas, para que adopten reales medidas de prevención y se logre disminuir la "cultura" del ocultamiento de los accidentes de trabajo.

El presente estudio, a diferencia de los otros, utilizó información de la fuente inicial, el Hospital General de Zona, donde se otorga la primera atención médica. Ésta es una aproximación más real al subregistro, en comparación con otras investigaciones, que solo tomaron una muestra de algunos meses de subregistro de los casos que llegaron a SST. Una limitación importante de los estudios previos son las fuentes de información de donde tomaron los casos, pues solo recopilaron los AT de los Servicios de Salud en el Trabajo (SST) que no concluyeron su trámite; otro aspecto es que los periodos de estudio fueron muy cortos, en los cuales no se confrontaron sus datos con las diferentes bases de datos del IMSS, como son los expedientes clínicos, la ST-5 (hoja para codificación de riesgos de trabajo ocurridos y terminados y casos de invalidez), que les permitieran identificar los factores que intervinieron en la noconclusión del tramite de calificación del AT.

Existen varios lineamientos involucrados en el proceso de la calificación de AT, así como aspectos que afectan la oportunidad de su emisión; aunque podemos identificarlos, no conocemos el peso específico de estos factores que están influyendo en el seguimiento de los casos, desde la primera atención hasta su resolución, tanto médica como legal del AT. El proceso de atención de un probable AT sigue una serie de rutas médicas y administrativas, en las cuales se puede generar un obstáculo para que el proceso se detenga y no concluya en un caso de AT terminado y se registre en el Sistema de Información de Riesgos de Trabajo. Estas rutas pueden incluir desde los casos en que el trabajador no regresa a los SST con la ST-1 requisitada, el HGZ no envía todos los formatos de ST-4-30-8 a los SST, hasta aquellos en que no se confrontan periódicamente los casos atendidos con ST-4-30-8 (nota de urgencias) con los accidentes de trabajo calificados y terminados (ST-5), lo que permitiría identificar los casos que deberán ser enviados al Departamento de Auditoría a patrones para recabar los datos de la empresa en cada AT, como se muestra en la figura 1.

Aunque en este estudio no pretendemos costear la atención del subregistro, calculamos que, de los 493 casos de la UMFX que recibieron al menos una consulta de urgencias y cuyo precio promedio es de $\$ 503.00$ pesos, ${ }^{18}$ si lo multiplicamos por los 493 casos, el costo total de atención de urgencias fue de $\$ 247,979.00$ pesos, sin tomar en cuenta los exámenes de laboratorio y gabinete, que no se registraron en el expediente ni en la nota de urgencias, ni los días de incapacidad o interconsultas.

\section{RECOMENDACIONES}

En el procedimiento de la atención de los accidentes de trabajo por parte de los servicios de urgencias de cualquier unidad o centro hospitalario del IMSS que otorguen la primera atención de un probable RT, deberían enviarse periódicamente los formatos de atención médica a cada una de las UMF correspondientes y a los Servicios de Salud en el 
Trabajo, lo que permitiría hacer un seguimiento de los casos de accidentes de trabajo hasta su conclusión; además, sería muy conveniente que estos servicios de urgencias conservaran una copia de estos formatos para su propio archivo.

Con los resultados de este estudio se encontró que no existe un control eficaz de los accidentes de trabajo durante todo el proceso de atención médica y calificación del riesgo; esto genera un subregistro de los casos de accidentes de trabajo, con el consecuente impacto en las finanzas de la institución, de los trabajadores y la falta de aplicación de la leyes en la materia. ${ }^{16,} 17$ Por lo anterior, sugerimos algunas medidas simples de intervención para poder reducir el subregistro y contribuir a disminuir el desfinanciamiento de la institución. Estas medidas son:

Por lo que se refiere a la Ley del Seguro Social, es necesario hacer las modificaciones pertinentes en los servicios de atención médica y la dictaminación del accidente de trabajo, que conduzcan a que la calificación del riesgo de trabajo se realice en la institución dentro de las primeras $24 \mathrm{hrs},{ }^{19}$ independientemente de quién requisite los datos de la ST-1.

En el caso de HGZ, consideramos que debe cumplir con la normatividad existente, la cual establece la obligatoriedad y su respectivo envío y notificación de los casos de probables accidentes de trabajo que recibieron atención médica a los SST de las unidades médicas de su influencia. En la UMF, los médicos familiares deben notificar a la brevedad a los SST de cualquier accidente de trabajo que sea atendido en la consulta externa, para que concluya el proceso de calificación del mismo.

Finalmente, consideramos que la Coordinación de Salud en el Trabajo, conjuntamente con los Servicios Salud en el Trabajo, debería elaborar una nueva propuesta del procedimiento de valoración de los accidentes de trabajo, la cual incluya la calificación oportuna y un sistema de control y seguimiento de los casos de accidente de trabajo desde la atención médica inicial, así como su subsiguiente registro en el Sistema Nacional de Información de accidentes de trabajo, ya que esto permitirá que la incidencia de riesgos esté contemplada de manera real en el cálculo de la prima de riesgos de trabajo que el IMSS debe recuperar de las empresas afiliadas.

\section{AGRADECIMIENTOS}

Agradecemos ampliamente la colaboración del personal de archivo del HGZ, al personal administrativo y médico del Servicio de Salud en el Trabajo de la UMF y de la Delegación No. 4 Suroeste del Distrito Federal. Finalmente, a las autoridades de todas las Unidades por las facilidades que nos otorgaron para el buen término del presente estudio. 


\section{BIBLIOGRAFÍA}

1. Panorama epidemiológico de la población económicamente activa, Unidad Temática: Salud en el Trabajo. Departamento de Salud Pública Enseñanza. http://www.facmed.unam/deptos/salud/strabajo/panorama.html. Consultado en enero del 2003

2. Salinas-Tovar, J.S.; López-Rojas, P.; SotoNavarro, M.O.; Caudillo-Araujo, D.E.; SánchezRomán, F.R.; Borja-Aburto, V.H. El subregistro potencial de los accidentes de trabajo en el Instituto Mexicano del Seguro Social. Salud Pública de México, 2004; 46: 204-209.

3. Memorias estadísticas 1985 a 2005. Coordinación de Salud en el Trabajo. División de prevención de riesgos de trabajo y área de promoción de los trabajadores. División Técnica de Información en Estadística en Salud. ST-5. Documento Interno Institucional. IMSS.

4. http://laborsta.ilo.org/cgi-bin/brokerv8.exe. Estadísticas de la Organización Internacional del Trabajo, 2003, Daños ocupacionales: Agrupa accidentes y enfermedades de trabajo no fatales. Consultado en enero 2002.

5. Jaramillo, M.A. Riesgos de trabajo no reclamados, Memorias, I Reunión Nacional de Investigación en Salud en el Trabajo, México, IMSS; 1997; Resumen, 29: 36.

6. Acosta Vega, J. Fernando; Sánchez Chacón, Georgina. Riesgos de trabajo no reclamados en la Delegación Guerrero del IMSS, zona Acapulco en 1996, II Reunión Nacional de Investigación en Salud en el Trabajo, México. IMSS. 1998; Resumen, 40: 75.

7. Macías, R.E.; Pérez, P.A. Seguimiento administrativo de los probables riesgos de trabajo atendidos en el servicio de Urgencias de adultos del HGR 46, Guadalajara, III Reunión Nacional de Investigación en Salud en el Trabajo, México, IMSS; 1999; Resumen, 41: 74.

8. Documentos de la Dirección Regional Siglo XXI, Delegación 4, México, IMSS, 2002. Documento Interno Institucional.

10. Sistema de Información Nacional de Dere- chos y Obligaciones, 2002, Sistema Institucional Interno. División de Afiliación y Registro de Beneficiarios, México, IMSS.

11. García, B.R.; Guerrero, L.A.; Becerra, V.R. Riesgos de trabajo no reclamados en la UMF 61 del periodo de enero a diciembre del 2000, VII Reunión Nacional de Investigación en Salud en el Trabajo, México, IMSS, 2003, Resumen 69.

12. Franco Enríquez, J.G.; Cuéllar Romero, R.; Noriega Elío, Ma.; Villegas Rodríguez, J. Los trabajadores textiles de Naucalpan, Estado de México, y sus condiciones de salud en 1989, Salud Problema. 1994;24:33-43.

13. Colunga, G.; Cervantes, R. Inconcluso trámite administrativo del reclamo de riesgos de trabajo, II Reunión Nacional de Investigación en Salud en el Trabajo. Memoria, México, IMSS, 1998, Resumen 41: 76.

14. Montes de Oca, M.C.; E.J.; Turcott, Dolores; López Rojas, Pablo. Factores asociados a la noreclamación de los riesgos de trabajo en la zona de Toluca, Estado de México, durante 1994, III Reunión Nacional de Investigación en Salud en el Trabajo, México, IMSS; 1999; Resumen 52: 86.

15. Montes De Oca, M.C.; Turcott Dolores, López Rojas, Pablo. Factores asociados en trabajadores que no reclamaron sus riesgos de trabajo en la zona Toluca, Estado de México durante 1995, I Reunión Nacional de Investigación en Salud en el Trabajo, México, IMSS; 1997; Resumen 30: 36.

16. Ley Federal del Trabajo, Artículos 477 y 480, del Seguro de Riesgos de Trabajo, Título noveno, México, Delma; 2002: 113.

17. Ley del Seguro Social, Sección segunda, artículos 56, y sección tercera, 58, 2003, México, Ediciones Fiscales ISEF.

18. Costos Unitarios de Atención Médica. 2002, Documento Institucional Interno. Coordinación de Presupuesto e Información Programática, México, IMSS.

19. Reglamento de Servicios Médicos, Capítulo II de la Atención Médica en el Seguro de Riesgos de Trabajo, Art. 21, 2003, México, Ediciones Fiscales ISEF. 